\title{
Rotura vesical extraperitoneal coincidiendo con esquirlas óseas: necesidad de tratamiento quirúrgico
}

\author{
Luis A. Fariña Pérez*, Daniel Pesqueira Santiago*, Pedro Posadilla Casquete**, \\ Carmen Trinidad López*** \\ *Servicios de Urología, ${ }^{* *}$ Traumatología y ${ }^{* * *}$ Radiología. Hospital POVISA, Vigo. Pontevedra, España.
}

\begin{abstract}
Resumen
Introducción: En las roturas vesicales traumáticas cerradas, se ha recomendado reparar quirúrgicamente la rotura intraperitoneal y tratar de manera conservadora la extraperitoneal, mediante sonda, antibióticos y comprobación del cierre de la herida vesical a las dos o tres semanas. Sólo recientemente, se ha prestado importancia a la existencia de esquirlas óseas de las fracturas pelvianas vecinas a la lesión vesical, que en presencia de una sonda permanente o de una orina infectada pueden dar lugar a fístulas persistentes o infecciones pelvianas graves.

Pacientes y Método: Los dos casos de rotura vesical extraperitoneal que presentamos ponen de manifiesto la dificultad del diagnóstico seguro mediante TAC, y la gravedad de la existencia de esquirlas óseas improntando en la disrupción de la vejiga.

Comentario: Los consensos recientes en traumatismo genitourinario destacan la necesidad de prestar atención a la presencia de esquirlas óseas de las facturas del anillo pelviano en los traumatismos vesicales cerrados. En caso de rotura vesical extraperitoneal con fragmentos óseos en la vecindad, la exploración endoscópica y la reparación quirúrgicas pueden ser necesarias.
\end{abstract}

Palabras clave: Rotura vesical. Trauma urológico.

\section{Extraperitoneal bladder rupture coinciding with bone fragments: the case for surgical treatment}

\section{Abstract}

Introduction: In closed traumatic bladder ruptures, a surgical treatment of the intraperitoneal rupture, and a conservative approach to the extraperitoneal one, with bladder catheter, antibiotics and confirmation of bladder wound closure after two to three weeks-, was recommended. Only recently, attention was paid to the importance of the presence of bone fragments of the pelvic fractures, that in the vecinity of a bladder catheter or urinary infection may give raise to either severe pelvic infection or permanent urinary fistula.

Patients and Method: The two cases of extraperitoneal bladder rupture here presented, demonstrate the difficulties of a secure diagnosis by CT, and the potential severity of the existence of bone fragments impronting the bladder disruption.

Comment: Recent consensus on genitourinary trauma emphasizes the need to pay attention to the presence of bone fragments of the pelvic ring fractures in cases of closed bladder rupture. In cases of extraperitoneal bladder rupture with bone fragments in the vecinity, bladder endoscopic examination and surgical treatment may be needed.

Keywords: bladder rupture. Urological trauma.

$\mathrm{E}$ las roturas vesicales traumáticas cerradas, clásicamente, se ha recomendado reparar quirúrgicamente la rotura intraperitoneal y tratar de manera conservadora la extraperitoneal, mediante sonda uretral, antibióticos y comprobación radiológica del cierre de la herida vesical a las dos o tres semanas. Sólo recientemente, se ha prestado importancia a la existencia de esquirlas óseas de las fracturas pelvianas vecinas a la rotura vesical, pues en presencia de una sonda permanente o de una orina infectada pueden dar lugar a fístulas persistentes o infecciones pelvianas graves.

Los dos casos que presentamos muestran la dificultad del diagnóstico seguro mediante TAC y cómo, cuando la rotura vesical extraperitoneal coincide con la vecindad de esquirlas óseas, la mejor opción es la reparación operatoria de las lesiones y el drenaje del espacio perivesical, si el paciente esté hemodinámicamente estable.

\section{MATERIAL Y METODO}

\section{Caso 1}

Mujer de 82 años, que ingresa tres días después de sufrir una caída casual en su domicilio, habiendo estado encamada en casa durante este tiempo. Se le encuentra fractura compleja del anillo pelviano, otras fracturas varias en extremidades e hiponatremia grave. En estudio de TAC, existía una rotura extraperitoneal de la vejiga coincidiendo con impronta de hueso isquion izquierdo, sin extravasado perivesical significativo (Fig. 1). Debido a la edad y al tiempo transcurrido desde el traumatismo, se optó por hacer tratamiento conservador de las fracturas, y sondaje permanente hasta cicatrización de la lesión vesical extraperitoneal, quedando a cargo del Servicio de Nefrología para compensar los trastornos electrolíticos. A los 25 días de hospitalización presenta episodios febriles y se encuentra un absceso inguinal izquierdo con trayecto fistuloso hasta la cara anterior 


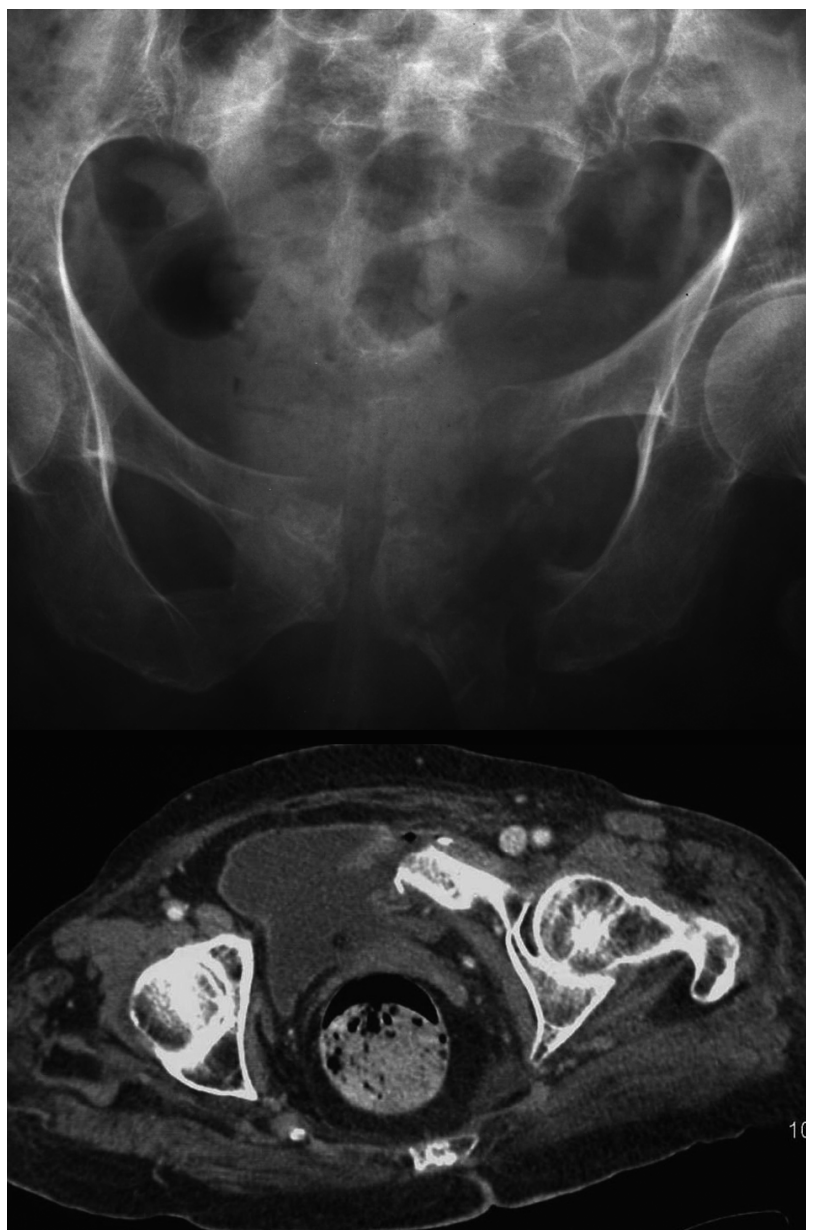

FIGURA 1. RX simple y TAC: fractura compleja del anillo pelviano, y rotura extraperitoneal de la vejiga coincidiendo con impronta de hueso isquion izquierdo, sin extravasado perivesical significativo.

del muslo, y un nuevo TAC muestra persistencia de la rotura vesical con fuga de contraste por el trayecto fistuloso, formando un absceso (Fig. 2). Se drenó el absceso y reparó quirúrgicamente la vejiga, con curación de las heridas sin secuelas.

\section{Caso 2}

Varón de 75 años que es encontrado accidentado en la vía pública, con trauma cráneo-encefálico, fracturas en extremidades superior e inferior derechas y costales, con enfisema subcutáneo intenso, y hematuria leve. Ingresa en la Unidad de Cuidados Intensivos para soporte ventilatorio y en los estudios de imagen de la pelvis se le encuentra fractura cerrada de huesos pelvianos con esquirla ósea improntando en la vejiga (Fig. 3). Una reconstrucción de las imágenes de TAC parecía descartar la lesión vesical penetrante (Fig. 4). Sin embargo, una vez estabilizadas las constantes vitales, se realiza cistoscopia que demuestra

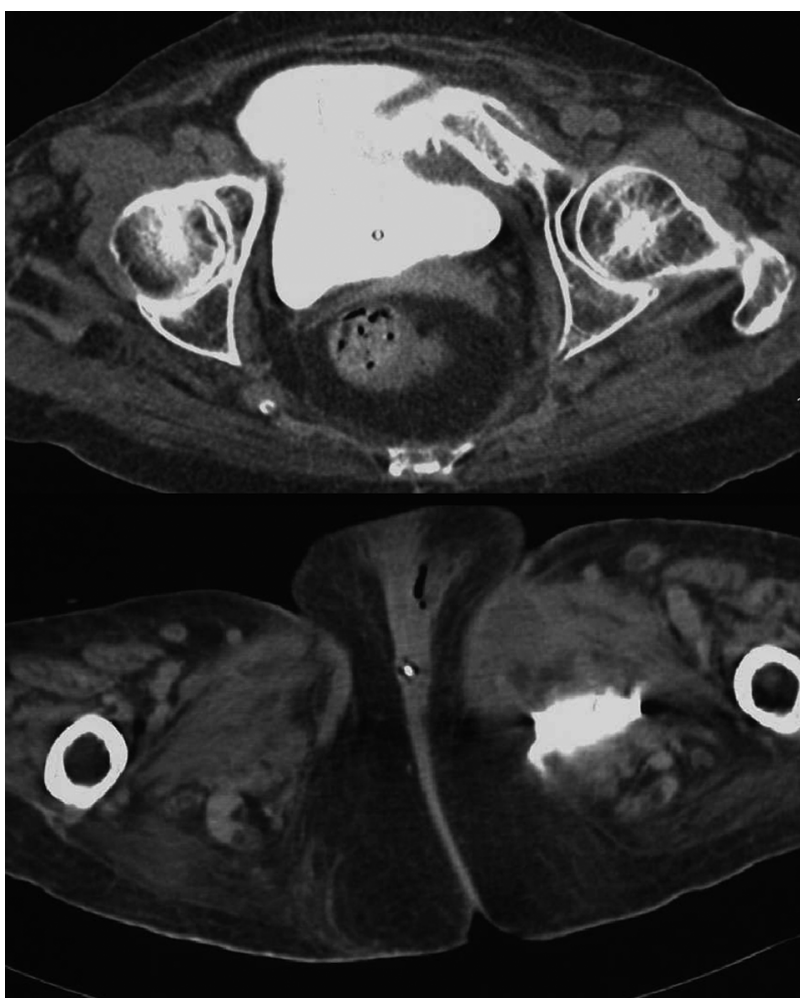

FIGURA 2. TAC de abdomen: se encuentra persistencia de rotura vesical con extravasado extraperitoneal y trayecto fistuloso hasta la cara anterior e interna del muslo izquierdo, formando un absceso.

perforación de la pared vesical anterior por la esquirla ósea. Por un acceso suprapúbico se reparó la vejiga y extirpó el fragmento óseo, sin secuelas y con posterior buena curación de las fracturas.

\section{DISCUSIÓN}

Incluso en centros con protocolos de traumatismo, las roturas vesicales pasan inadvertidas hasta en un 25\% de los pacientes, con retrasos diagnósticos de casi veinticuatro horas para las lesiones intraperitoneales y de hasta 7 días para las extraperitoneales ${ }^{1}$. Uno de las causas de este retraso es el estudio de imagen subóptimo, en el que la vejiga no se rellena retrógradamente hasta un volumen suficiente para demostrar la lesión (cistografía-TC con distensión vesical máxima). Un examen de TC con contraste endovenoso antes y después de micción, cuando es posible hacerlo, o bien después de evacuar la vejiga con sonda, puede suplir con cierta garantía a la cistografía de relleno o a la cistografía$\mathrm{TC}^{2-4}$. Una complicación reconocida de la rotura vesical extraperitoneal inadvertida es la infección séptica del espacio pelviano, que de forma no infrecuente tiene extraordinaria gravedad, como en la 


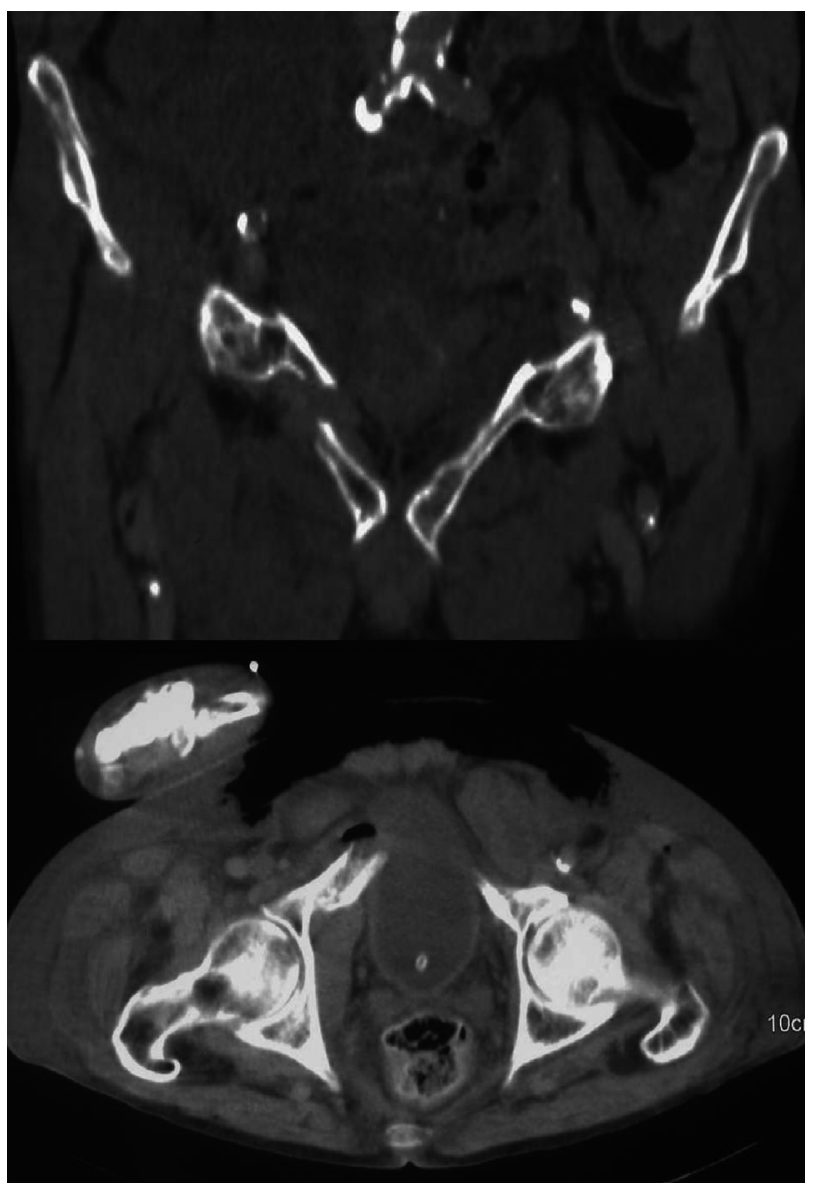

FIGURA 3. TAC de abdomen, reconstrucción coronal y corte axial: fractura del anillo pélvico en el lado derecho, sin extravasado perivesical. Obsérvese el intenso enfisema subcutáneo en la cara anterior del abdomen, consecuencia de fracturas costales.

primera paciente nuestra, con una rotura tratada precozmente con sonda permanente y antibióticos, pero que se complicó ulteriormente con infección pelviana y fístula urinaria exteriorizada en el muslo.

En los últimos años prevaleció la recomendación general de tratar la lesión vesical extraperitoneal de manera conservadora, manteniendo una sonda y antibióticos hasta la curación demostrada de las lesiones $^{5,6}$, pero esta forma de tratamiento no está exenta de riesgos y de fracasos ${ }^{7}$, en particular en roturas extensas, cuando afectan al cuello vesical o cuando coinciden con la vecindad de esquirlas óseas. En estos casos es mejor opción la reparación operatoria de las lesiones y el drenaje del espacio perivesical, cuando el paciente esté hemodinámicamente estable, tal como se recomienda en protocolos más recientes ${ }^{8,9}$.

Los traumatismos vesicales en ocasiones se asocian a lesiones óseas, viscerales o vasculares de tal

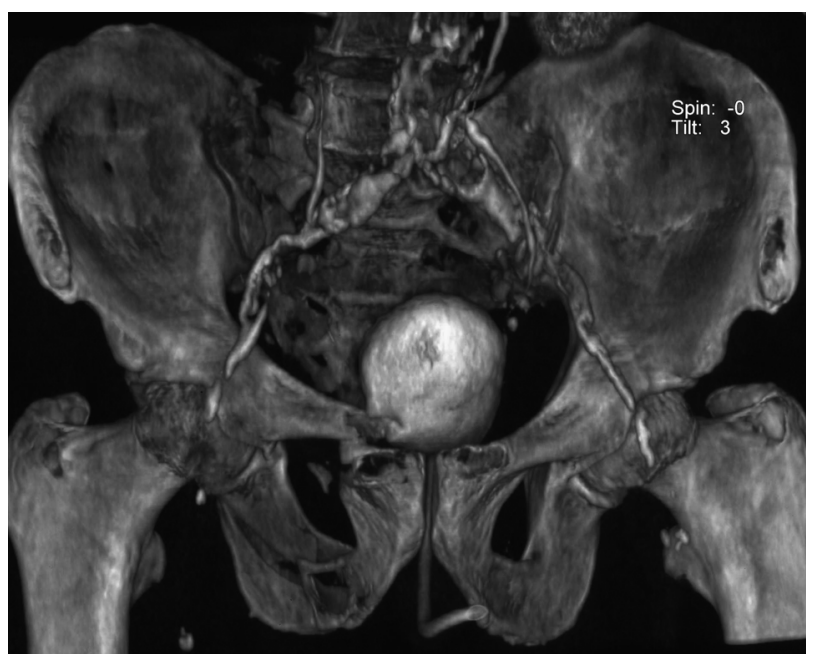

FIGURA 4. Reconstrucción tridimensional de las imágenes de TAC, que parece descartar la lesión vesical penetrante (hay una esquirla ósea improntando la pared vesical anterior- derecha, pero no hay extravasado de contraste).

gravedad, que la atención urológica pasa a un segundo plano o es diferida durante días o semanas. Teniendo en cuenta que la mortalidad de estos pacientes sigue siendo muy alta, generalmente a consecuencia de esos daños asociados, es preciso procurar que las lesiones urológicas no contribuyan a estas cifras aún tan dramáticamente elevadas.

\section{REFERENCIAS}

1. Ziran BH, Chamberlin E, Shuler FD, Shah M. Delays and difficulties in the diagnosis of lower urologic injuries in the context of pelvic fractures. J Trauma 2005;58(3):533-537

2. Haas CA, Brown SL, Spirnak JP. Limitations of routine spiral computerized tomography in the evaluation of bladder trauma. J Urol. 1999; 162(1):51-2.

3. Horstman WG, McClennan BL, Heiken JP. Comparison of computed tomography and conventional cystography for detection of traumatic bladder rupture. Urol Radiol 1991;12(4):188-193.

4. Kane NM, Francis IR, Ellis JH. The value of CT in the detection of bladder and posterior urethral injuries. AJR 1989;153(6): 1243-6.

5. Cass AS, Luxenberg M. Management of extraperitoneal ruptures of bladder caused by external trauma. Urology 1989;33(3):179-83.

6. Corriere JN Jr, Sandler CM. Diagnosis and management of bladder injuries. Urol Clin North Am 2006;33(1):67-71.

7. Kotkin L, Koch MO. Morbidity associated with nonoperative management of extraperitoneal bladder injuries. J Trauma 1995;38(6):895-898.

8. Lynch D, Martínez-Piñeiro L, Plas E et al. Urological trauma, p 34. En European Association of Urology guidelines. European Association of Urology, Arnheim, 2006.

9. Gómez RG, Ceballos L, Coburn M, et al. Consensus statement on bladder injuries. BJU Int 2004;94(1):27-32.

Correspondencia autor: Dr. Luis A. Fariña

Servicio de Urología. Hospital POVISA

Salamanca 5 - 36211 Vigo, Pontevedra

Tel.: 986413144

E-mail autor: luisfarina@yahoo.com

Información: Nota clínica

Trabajo recibido: junio 2007

Trabajo aceptado: septiembre 2007 